\title{
On Optimal Input Design in System Identification for Control
}

\author{
Bo Wahlberg, Håkan Hjalmarsson and Mariette Annergren
}

\begin{abstract}
This paper considers a recently proposed framework for experiment design in system identification for control. We study model based control design methods, such as Model Predictive Control, where the model is obtained by means of a prediction error system identification method. The degradation in control performance due to uncertainty in the model estimate is specified by an application cost function. The objective is to find a minimum variance input signal, to be used in system identification experiment, such that the control application specification is guaranteed with a given probability when using the estimated model in the control design. We provide insight in the potentials of this approach by finite impulse response model examples, for which it is possible to analytically solve the optimal input problem. The examples show how the control specifications directly affect the excitation conditions in the system identification experiment.
\end{abstract}

\section{INTRODUCTION}

We will study optimal input signal design for system identification when the estimated models are used in model based control. This work is motivated by a recent framework presented in [13] that in a direct way connects the system identification experimental conditions and the control performance. The identification objective is to guarantee, with a given probability, that the estimated model belongs to the set of models that satisfies the control specifications. System identification for control has been an active area of research for many years, see [15] for an overview. Recently, there has been extensive progress in optimal experiment design, [22], [5], [18], [3], [17], [26], [24], [6], [23], [1]. The key idea is to convexify this type of optimization problems, see [12], [16], [2], [4], [14], [8].

Consider a scalar discrete time asymptotically stable timeinvariant linear dynamical system with impulse response sequence $\left\{g_{k}\right\}$, input signal sequence $\{u(t)\}$, output signal sequence $\{y(t)\}$, and additive zero mean white Gaussian noise $\{e(t)\}$ with variance $\lambda_{e}$. We then have

$$
y(t)=\sum_{k=1}^{\infty} g_{k} u(t-k)+e(t), \quad t=1,2, \ldots
$$

The objective is to design a model based controller for the system (1), e.g., to reject disturbances. We will study parametric models, including black-box models such as ARX, FIR or Box-Jenkins models. Introduce the model parameter vector $\theta \in \mathbb{R}^{n}$, and assume that the true system can be described by the parameters in $\theta_{o}$. Define the application cost function $V_{a p p}(\theta)$ that measures the degradation in control performance for a certain model based control application if we have errors in the system parameters. Without loss of generality, we can assume that $V_{a p p}(\theta) \geq 0$ with minimum $V_{\text {app }}\left(\theta_{o}\right)=0$.

Let $\hat{\theta}_{N}$ be a parameter vector estimate obtained from $N$ measured input output observations from the system and

This work was partially supported by the Swedish Research Council and the Linnaeus Center ACCESS at KTH

Automatic Control Lab and ACCESS, School of Electrical Engineering, KTH, SE-100 44 Stockholm, Sweden. (e-mail: bo.wahlberg@ee.kth.se, hakan.hjalmarsson@ee.kth.se.) using PEM system identification, see [20]. The performance index $V_{a p p}\left(\hat{\theta}_{N}\right)$ measures the control performance when the model corresponding to $\hat{\theta}_{N}$ is used in the control design of the application, e.g., Model Predictive Control (MPC) or Internal Model Control. The Cramér-Rao lower bound, see [20], translates into lower bounds on the achievable accuracy of system properties that are functions of the model parameters. It also implies a fundamental performance bound for model based applications employing identified models:

$$
\begin{aligned}
\lim _{N \rightarrow \infty} N \mathrm{E}\left[V_{a p p}\left(\hat{\theta}_{N}\right)\right] & \geq \frac{1}{2} \text { Trace }\left\{V_{a p p}^{\prime \prime}\left(\theta_{o}\right) \mathbf{I}_{F}^{-1}\right\}, \\
\mathbf{I}_{F} & =\frac{1}{2 \pi} \int_{-\pi}^{\pi} \Psi\left(\mathrm{e}^{i \omega}\right) \Psi^{*}\left(\mathrm{e}^{i \omega}\right) \mathrm{d} \omega,
\end{aligned}
$$

where $\Psi(z)$ in $\mathbf{I}_{F}$ (the average Fisher information matrix) is the (normalized) prediction error gradient. We use prime to denote differentiation, and $V_{a p p}^{\prime \prime}\left(\theta_{o}\right)$ is the Hessian of $V_{a p p}(\theta)$ at $\theta=\theta_{o}$.

The bound (2) contains information on how experiment configuration (input excitation etc), system and model complexity, and model structure, as well as the property of interest (through the performance index $V_{a p p}(\theta)$ ), influence performance. However, much due to the matrix inverse of $\mathbf{I}_{F}$, it is non trivial to analyze these dependencies and often extensive matrix manipulations are required. Understanding the nature of (2) has been subject to intense research for a range of problem settings, including frequency function estimation [19], multi-input identification [9], and Hammerstein systems [21]. For linear time invariant (LTI) singleinput single-output systems, it has been shown in [25] that an invariant, or water-bed effect, holds for the asymptotic (large $N$ ) variance of the frequency response function estimate $\operatorname{As} \operatorname{Var} G\left(e^{i \omega}, \hat{\theta}_{N}\right)$. For output error models this effect can be expressed as

$$
\frac{1}{2 \pi} \int_{-\pi}^{\pi} \operatorname{As} \operatorname{Var} G\left(e^{i \omega}, \hat{\theta}_{N}\right) \Phi_{u}(\omega) d \omega=\frac{\lambda_{e} n}{N},
$$

where $\Phi_{u}(\omega)$ is the input power spectral density, $\lambda_{e}$ is the measurement noise variance, and $n$ is the number of estimated parameters. This invariance result shows that the quality and shape of the model error is only determined by the spectral properties of the input signal. The solution of the input optimization problem to be studied will be the optimal power spectral density function $\Phi_{u}(\omega)$ or the corresponding covariance function

$$
r_{\tau}=\mathrm{E}\{u(t) u(t-\tau)\}=\frac{1}{2 \pi} \int_{-\pi}^{\pi} \Phi_{u}(\omega) e^{i \omega \tau} d \omega .
$$

\section{Application Sets for System IdentificAtion INPUT DESIGN}

Building on [4], Hjalmarsson and co-workers have recently developed a framework for experiment design, see [13]. Let, as before, $V_{a p p}(\theta)$ denote the application performance cost function when the model parameter $\theta$ is used in 
the model based design. The specification of the application is given by

$$
V_{\text {app }}(\theta) \leq \frac{1}{2 \gamma},
$$

for some constant $\gamma>0$. We assume that $V_{a p p}(\theta) \geq 0$, and $V_{a p p}\left(\theta_{o}\right)=0$ for the true parameter. The specification (5) implies that the estimated parameter vector $\hat{\theta}_{N}$ must end up in the set $\left\{\theta: V_{a p p}(\theta) \leq 1 /(2 \gamma)\right\}$ with a given probability. Since $V_{a p p}(\theta) \geq 0$ with minimum $V_{a p p}\left(\theta_{o}\right)=0$, we have the approximation

$$
\begin{aligned}
V_{a p p}(\theta) & \approx V_{a p p}\left(\theta_{o}\right)+V_{a p p}^{\prime}\left(\theta_{o}\right)\left[\theta-\theta_{o}\right] \\
& +0.5\left[\theta-\theta_{o}\right]^{T} V_{a p p}^{\prime \prime}\left(\theta_{o}\right)\left[\theta-\theta_{o}\right] \\
& =0+0.5\left[\theta-\theta_{o}\right]^{T} V_{a p p}^{\prime \prime}\left(\theta_{o}\right)\left[\theta-\theta_{o}\right],
\end{aligned}
$$

where we have neglected higher order terms. This means that the application specifications (5) can be approximated by the ellipsoidal set

$$
\mathscr{E}_{a p p}=\left\{\theta:\left[\theta-\theta_{o}\right]^{T} V_{a p p}^{\prime \prime}\left(\theta_{o}\right)\left[\theta-\theta_{o}\right] \leq \frac{1}{\gamma}\right\} .
$$

The quality of this approximation depends on $V_{a p p}(\theta)$, but also on $\gamma$. A high value of $\gamma$ gives a more restricted set and a better quadratic approximation.

A key result in prediction error/maximum likelihood system identification, see [20], is the asymptotic (large $N$ ) quality property

$$
\hat{\theta}_{N} \in \mathscr{E}_{S I}=\left\{\theta:\left[\theta-\theta_{o}\right]^{T} \mathbf{I}_{F}\left[\theta-\theta_{o}\right] \leq \frac{\kappa}{N}\right\}, \quad \text { w.p. } \alpha,
$$

where $\mathbf{I}_{F}$ is the average Fisher information, see (3). The constant $\kappa$ depends on the number of estimated parameters and the probability $\alpha$, and can be determined from the $\chi^{2}$ distribution. This result implies that we guarantee, with probability $\alpha$, that the estimated model belongs to the ellipsoidal set given by (7).

To connect system identification to the intended application we need to guarantee that the system identification set defined by (7) is inside the application set defined by the quadratic form (6). Since both sets are ellipsoidal, it is sufficient to verify the matrix inequality ${ }^{1}$

$$
\frac{N}{\kappa} \mathbf{I}_{F} \geq \gamma V_{a p p}^{\prime \prime}\left(\theta_{o}\right) \text {. }
$$

This inequality together with (7) imply

$$
1 \geq\left[\hat{\theta}_{N}-\theta_{o}\right]^{T} \frac{N}{\kappa} \mathbf{I}_{F}\left[\hat{\theta}_{N}-\theta_{o}\right] \geq \gamma\left[\hat{\theta}_{N}-\theta_{o}\right]^{T} V_{a p p}^{\prime \prime}\left(\theta_{o}\right)\left[\hat{\theta}_{N}-\theta_{o}\right]
$$

and hence $\hat{\theta}_{N} \in \mathscr{E}_{a p p},(6)$, with at least probability $\alpha$,

Two interesting observations can be made from (8):

i). The estimation properties (the left-hand side of (8)) are separated from the application specifications (the righthand side).

ii). The inequality is in terms of $\mathbf{I}_{F}$ rather than its inverse (as in (2)). This opens up for both simple and efficient numerical methods in optimal experiment design.

\footnotetext{
${ }^{1} X \geq Y$ means that $[X-Y]$ is a positive semi-definite matrix.
}

We will study the following minimum variance input design problem, which is related to least costly identification experiment design introduced in [4],

$$
\min _{u(t)} \mathrm{E}\left\{u^{2}(t)\right\} \quad \text { s.t. } \quad \frac{N}{\kappa} I_{F} \geq \gamma V_{a p p}^{\prime \prime}\left(\theta_{o}\right)
$$

where $\{u(t)\}$ is the input signal to be used in the system identification experiment. This means that we want to find the minimum power input signal such that the application specification is satisfied for the estimated model $\hat{\theta}_{N}$. A remaining issue is to understand how the Fisher information matrix $\mathbf{I}_{F}$ depends on the input signal. In general, $\mathbf{I}_{F}$ is an affine function in the input power spectral density $\Phi_{u}(\omega)$ and since

$$
\mathrm{E}\left\{u^{2}(t)\right\}=\frac{1}{2 \pi} \int_{-\pi}^{\pi} \Phi_{u}(\omega) d \omega
$$

is linear in $\Phi_{u}(\omega)$ we obtain a Semi-Definite Program (SDP) (linear cost-function and linear matrix inequity constraint) where we optimize with respect to the power spectral density or the corresponding covariance function. This infinite dimensional convex optimization problem can be approximately solved using a finite dimensional parametrization of $\Phi(\omega) \geq 0$. The problems simplifies greatly for a Finite Impulse Response (FIR) model of order $(n-1)$, since $\mathbf{I}_{F} / \lambda_{e}$ will be a Toeplitz matrix with the first $n$ values of the covariance function of $\{u(t)\}$ as first column. Since the cost function is the variance of $\{u(t)\}$, we obtain a SDP only involving $r_{\tau}=\mathrm{E}\{u(t) u(t-\tau)\}, \tau=0, \ldots,(n-1)$.

\section{A. Examples of Control Application Sets}

We will start by studying application sets for a certain simple control problem. Our objective is not to optimally solve a specific control problem, but rather to illustrate the basic concepts and provide insights into the optimal input solutions.

Consider the first order FIR system $(n=2)$

$$
y(t)=b_{1}^{o} u(t-1)+b_{2}^{o} u(t-2)+d(t)
$$

with input signal sequence $\{u(t)\}$, output signal sequence $\{y(t)\}$, and disturbance $\{d(t)\}$. The control objective is to reject the disturbance $d(t)$, i.e., to keep $y(t)$ close to zero. To simplify the calculations we will assume $d(t)$ to be a unit step disturbance,

$$
d(t)=\left\{\begin{array}{ll}
0, & t<0 \\
1, & t \geq 0
\end{array} .\right.
$$

We will first study the steady state error under the assumption that the true static gain is positive, $b_{1}^{o}+b_{2}^{o}>0$. The feed forward open loop controller to this disturbance rejection problem is $u(t)=-d(t) /\left(b_{1}+b_{2}\right)$. We will study the effects of errors in the static gain $b_{1}^{o}+b_{2}^{o}$ on the steady state control error using the application cost function

$$
\begin{aligned}
V_{a p p}(\theta) & =\left[1-\frac{b_{1}^{0}+b_{2}^{0}}{b_{1}+b_{2}}\right]^{2}, \quad \theta=\left[b_{1} b_{2}\right]^{T} . \\
V_{a p p}\left(\theta_{o}\right) & =0, V_{a p p}^{\prime}\left(\theta_{o}\right)=0, V_{a p p}^{\prime \prime}\left(\theta_{o}\right)=\frac{2}{\left(b_{1}^{o}+b_{2}^{o}\right)^{2}}\left[\begin{array}{ll}
1 & 1 \\
1 & 1
\end{array}\right] .
\end{aligned}
$$

For small parameter variations around $\theta_{o}$ the specification $V_{a p p}(\theta) \leq 1 /(2 \gamma)$ can be approximated by an ellipsoidal set, 
c.f. (6):

$$
\begin{aligned}
V_{a p p}(\theta) & \approx\left[\theta-\theta_{o}\right]^{T}\left[\frac{1}{\left(b_{1}^{o}+b_{2}^{o}\right)^{2}}\left[\begin{array}{ll}
1 & 1 \\
1 & 1
\end{array}\right]\right]\left[\theta-\theta_{o}\right] \\
& =\frac{\left[\left(b_{1}+b_{2}\right)-\left(b_{1}^{o}+b_{2}^{o}\right)\right]^{2}}{\left(b_{1}^{o}+b_{2}^{o}\right)^{2}} \leq \frac{1}{2 \gamma} .
\end{aligned}
$$

Note that we only have to worry about the accuracy of the static gain $b_{1}+b_{2}$. This could be seen already from (12). For this problem the solution to (9) is a constant signal, see (20). We have to use the concept of quasi-stationarity, [20], to define the corresponding covariance function.

A more interesting case is to use a feedback P-regulator $u(t)=-K y(t)$ to reduce the influence of $d(t)$ in $y(t)$. The the static relation under feedback is

$$
y=\frac{d}{1+K\left(b_{1}^{0}+b_{2}^{0}\right)} .
$$

To obtain a small static error the controller gain $K>0$ should be taken as large as possible. For $\left|b_{2}^{o} / b_{1}^{o}\right|<1$ (zero inside the unit disc), the closed loop stability condition is $K<1 /\left(b_{1}^{o}-\right.$ $b_{2}^{o}$ ). By using the controller gain

$$
K=\frac{\beta^{2}}{\beta b_{1}-b_{2}}, \quad 0<\beta \leq 1,
$$

we obtain nominal closed loop poles in $-\beta$ and $-\beta b_{2} /\left(\beta b_{1}-b_{2}\right)$. Consider the application cost function

$$
V_{a p p}(\theta)=\left[F\left(\beta b_{1}-b_{2}\right)-F\left(\beta b_{1}^{o}-b_{2}^{o}\right)\right]^{2},
$$

where the performance related function $F(x)$ is at least two times differentiable. Here we stress that the performance of the feedback system depends only, through the controller gain $K$, on the model parameter linear relation $\beta b_{1}-b_{2}$. Examples of $F(x)$ are the static gain:

$$
F(x)=\frac{x}{x+\beta^{2}\left(b_{1}^{o}+b_{2}^{o}\right)},
$$

and closed loop poles:

$$
F(x)=-\frac{\beta^{2} b_{1}^{o}}{2 x} \pm \sqrt{\frac{\beta^{4}\left(b_{1}^{o}\right)^{2}}{4 x^{2}}-\frac{\beta^{2} b_{2}^{o}}{x}} .
$$

More general application functions are discussed at the end of this section. For (15)

$$
\begin{aligned}
V_{a p p}^{\prime}(\theta) & =2\left[F\left(\beta b_{1}-b_{2}\right)-F\left(\beta b_{1}^{o}-b_{2}^{o}\right)\right] F^{\prime}\left(\beta b_{1}-b_{2}\right)\left[\begin{array}{c}
\beta \\
-1
\end{array}\right], \\
V_{a p p}^{\prime \prime}\left(\theta_{o}\right) & =2 F^{\prime}\left(\beta b_{1}-b_{2}\right)\left[\begin{array}{c}
\beta \\
-1
\end{array}\right] \times\left[\begin{array}{c}
\beta \\
-1
\end{array}\right]^{T} F^{\prime}\left(\beta b_{1}-b_{2}\right) \\
& =2\left[F^{\prime}\left(\beta b_{1}^{o}-b_{2}^{o}\right)\right]^{2}\left[\begin{array}{cc}
\beta^{2} & -\beta \\
-\beta & 1
\end{array}\right] .
\end{aligned}
$$

The eigenvalues of the matrix $V_{a p p}^{\prime \prime}\left(\theta_{o}\right)$ equal $\beta^{2}+1$ (with eigenvector $[-\beta, 1]^{T}$ of size $\sqrt{1+\beta^{2}}$ ) and 0 (with eigenvector $[1, \beta]^{T}$ of size $\sqrt{1+\beta^{2}}$ ). If

$$
\left[\begin{array}{l}
b_{1} \\
b_{2}
\end{array}\right]=\left[\begin{array}{l}
b_{1}^{o} \\
b_{2}^{o}
\end{array}\right]+\delta\left[\begin{array}{l}
1 \\
\beta
\end{array}\right]
$$

then $\beta b_{1}-b_{2}=\beta b_{1}^{0}-b_{2}^{0}$ for all $\delta$ and $V_{a p p}\left(b_{1}, b_{2}\right)=0$. We can thus tolerate large errors in the zero eigenvalue direction. If instead

$$
\left[\begin{array}{l}
b_{1} \\
b_{2}
\end{array}\right]=\left[\begin{array}{l}
b_{1}^{o} \\
b_{2}^{o}
\end{array}\right]+\delta\left[\begin{array}{c}
-\beta \\
1
\end{array}\right]
$$

then $\beta b_{1}-b_{2}=\beta b_{1}^{o}-b_{2}^{o}-\delta\left(\beta^{2}+1\right)$. This means that

$$
V_{a p p}(\theta) \approx\left[\delta\left(\beta^{2}+1\right) F^{\prime}\left(\beta b_{1}^{o}-b_{2}^{o}\right)\right]^{2}
$$

which limits how much error we can tolerate in the large eigenvalue direction.

We can analytically solve the optimal SI input design problem (9) for this problem, see (20). The optimal input signal can be realized by an AR-process: $u(t)=-a u(t)+$ $e_{u}(t)$ with $a=\beta$. This shows a nice connection between the SI excitation signal and the closed loop dynamics. We need to increase the band-width of the excitation signal as the gain of the controller increases. The limiting case $\beta=1$ corresponds to the optimal input $u(t)=C \cos (\pi t)$. This result will be proved in Section II-B.

The P-controller $K=4 b_{2} / b_{1}^{2}$ gives a nominal closed loop double pole in $-2 b_{2} / b_{1}$. For nominal closed loop stability we assume that the zero of the system satisfies $\left|b_{2}^{o} / b_{1}^{o}\right|<0.5$. What is the sensitivity of the corresponding closed loop system? Using the same calculations as above with the application cost function

$$
V_{a p p}(\theta)=\left[\bar{F}\left(\frac{b_{2}}{b_{1}^{2}}\right)-\bar{F}\left(\frac{b_{2}^{o}}{\left(b_{1}^{o}\right)^{2}}\right)\right]^{2},
$$

leads to

$$
V_{a p p}^{\prime \prime}\left(\theta_{o}\right)=2\left[\bar{F}^{\prime}\left(\frac{b_{2}^{o}}{\left(b_{1}^{o}\right)^{2}}\right)\right]^{2}\left[\begin{array}{cc}
1 & \frac{2 b_{2}^{o}}{b_{1}^{o}} \\
\frac{2 b_{2}^{o}}{b_{1}^{o}} & 4 \frac{\left(b_{2}^{o}\right)^{2}}{\left(b_{1}^{o}\right)^{2}}
\end{array}\right] .
$$

The function $\bar{F}(x)$ should also here reflect some performance related quantity, e.g., the static gain. For this control problem it is important to estimate the linear combination $b_{1}-2\left[b_{2}^{o} / b_{1}^{o}\right] b_{2}$ accurately. The optimal input for system identification can be obtained using the AR process $u(t)=$ $-a u(t)+e_{u}(t)$ with $a=-2 b_{2}^{o} / b_{1}^{o}$. Notice that also here the AR pole equals the nominal closed loop pole!

For general nonlinear least squares problems

$$
V_{a p p}(\boldsymbol{\theta})=\sum_{k} V_{a p p, k}(\boldsymbol{\theta}), \quad V_{a p p, k}(\boldsymbol{\theta})=\left[F_{k}(\boldsymbol{\theta})-F_{k}\left(\boldsymbol{\theta}_{o}\right)\right]^{2}
$$

we need to sum up the Hessians $V_{a p p, k}^{\prime \prime}(\theta)$ to form $V_{a p p}^{\prime \prime}(\theta)$. It is often a good idea to use relative errors

$$
V_{a p p, k}(\theta)=\left[\frac{F_{k}(\theta)-F_{k}\left(\theta_{o}\right)}{F_{k}\left(\theta_{o}\right)}\right]^{2}, \quad F_{k}\left(\theta_{o}\right) \neq 0,
$$

which introduce uniform weighting of the terms in $V_{a p p}^{\prime \prime}(\theta)$. In case it is not possible to analytically calculate the Hessian, numerical differentiation can be used. Another approach is to fit a quadratic function to values of $V_{a p p}(\theta)$ in the neighborhood of $\theta=\theta_{0}$.

\section{B. The System Identification Set}

Given the measurements $\{u(t), y(t), t=1 \ldots N\}$, a prediction error system identification method finds the parameter estimate by minimizing a nonlinear least squares cost function

$$
\hat{\theta}_{N}=\arg \min _{\theta} V_{S I, N}(\theta) .
$$

The asymptotic $(N \rightarrow \infty)$ cost function is denoted by $V_{S I}(\theta)$ and is assumed to satisfy $V_{S I}\left(\theta_{o}\right)=\lambda_{e}$ and $V_{S I}^{\prime}\left(\theta_{o}\right)=0$, 
i.e. the true parameter vector minimizes the asymptotic cost function. The Fisher information matrix in (7) equals

$$
\mathbf{I}_{F}=\frac{1}{2 \lambda_{e}} V_{S I}^{\prime \prime}\left(\theta_{o}\right)
$$

To illustrate the corresponding theory reconsider the FIR system (10), without disturbance $d(t)$, but with additive white measurements noise with variance $\lambda_{e}$. The least squares cost function is

$$
\begin{aligned}
V_{S I, N}(\theta) & =\frac{1}{N} \sum_{t=3}^{N}\left[y(t)-b_{1} u(t-1)-b_{2} u(t-2)\right]^{2} \\
V_{S I, N}^{\prime}(\theta) & =\frac{-2}{N} \sum_{t=3}^{N}\left[y(t)-b_{1} u(t-1)-b_{2} u(t-2)\right]\left[\begin{array}{l}
u(t-1) \\
u(t-2)
\end{array}\right] \\
V_{S I, N}^{\prime}\left(\theta_{0}\right) & =\frac{-2}{N} \sum_{t=3}^{N}\left[\begin{array}{l}
u(t-1) \\
u(t-2)
\end{array}\right] e(t) \\
V_{S I, N}^{\prime \prime}\left(\theta_{0}\right) & =\frac{2}{N} \sum_{t=3}^{N}\left[\begin{array}{l}
u(t-1) \\
u(t-2)
\end{array}\right]\left[\begin{array}{l}
u(t-1) \\
u(t-2)
\end{array}\right]^{T} \\
V_{S I, N}^{\prime \prime}\left(\theta_{0}\right) & \rightarrow V_{S I}^{\prime \prime}\left(\theta_{0}\right)=2 \mathbf{R}, \quad N \rightarrow \infty \\
\mathbf{R} & =\left[\begin{array}{ll}
r_{0} & r_{1} \\
r_{1} & r_{0}
\end{array}\right], \quad r_{\tau}=\mathrm{E}\{u(t) u(t-\tau)\}
\end{aligned}
$$

Here we have assumed that $\{u(t)\}$ is a quasi-stationary sequence with covariance function $r_{\tau}$. Since $e(t)$ is assumed to be white Gaussian noise it follows that $0.5 V_{S I, N}^{\prime}\left(\theta_{0}\right)$ is Gaussian distributed with asymptotic covariance matrix $\left(\lambda_{e} / N\right) \mathbf{R}$. Using (16) it follows that $\hat{\theta}_{N}-\theta_{N}$ is normal distributed with zero mean and asymptotic (large $N$ ) covariance matrix $\left(\lambda_{e} / N\right) \mathbf{R}^{-1}$. Hence, $N / \lambda_{e}\left[\theta-\theta_{0}\right]^{T} \mathbf{R}\left[\theta-\theta_{0}\right]$ is $\chi^{2}$ distributed with two degrees of freedom. This means that we can find $\kappa$ such that $\hat{\theta} \in \mathscr{E}_{V S I}$ with probability $\alpha$, c.f. (7), where

$$
\mathscr{E}_{S I}=\left\{\theta:\left[\theta-\theta_{0}\right]^{T} \mathbf{R}\left[\theta-\theta_{0}\right] \leq \frac{\lambda_{e} \kappa}{N}\right\} .
$$

\section{Optimal InPUT DeSign for CONTROL RELEVANT} SYSTEM IDENTIFICATION

We will now in more detail study the "least costly" SI input design optimization problem

$$
\min _{r_{0}, r_{1}} r_{0} \quad \text { s.t. } \quad \frac{N}{\lambda_{e} \kappa}\left[\begin{array}{ll}
r_{0} & r_{1} \\
r_{1} & r_{0}
\end{array}\right] \geq \gamma V_{a p p}^{\prime \prime}\left(\theta_{o}\right),
$$

which is a convex optimization problem (SDP) with a linear matrix inequality constraint and linear cost function. The Toeplitz matrix $\mathbf{R}$ can be factorized as

$$
\left[\begin{array}{ll}
r_{0} & r_{1} \\
r_{1} & r_{0}
\end{array}\right]=\frac{1}{\sqrt{2}}\left[\begin{array}{cc}
1 & 1 \\
1 & -1
\end{array}\right]\left[\begin{array}{cc}
r_{0}+r_{1} & 0 \\
0 & r_{0}-r_{1}
\end{array}\right] \frac{1}{\sqrt{2}}\left[\begin{array}{cc}
1 & 1 \\
1 & -1
\end{array}\right] .
$$

Hence, the eigenvectors are independent of $r_{0}$ and $r_{1}$, while the eigenvalues are $r_{0}+r_{1}$ and $r_{0}-r_{1}$. For a given

$$
V_{a p p}^{\prime \prime}\left(\theta_{o}\right)=\left[\begin{array}{ll}
v_{1} & v_{2} \\
v_{2} & v_{3}
\end{array}\right] \geq 0,
$$

it is possible to analytically solve Problem (18). The determinant conditions for $\mathbf{R}-V_{a p p}^{\prime \prime}\left(\theta_{o}\right) \geq 0$ for a two by two matrix, see [7], is

$$
\begin{aligned}
\left(r_{0}-v_{1}\right) \geq 0, \quad\left(r_{0}-v_{3}\right) & \geq 0, \\
\left(r_{0}-v_{1}\right)\left(r_{0}-v_{3}\right)-\left(r_{1}-v_{2}\right)^{2} & \geq 0 .
\end{aligned}
$$

We see that $r_{1}=v_{2}$ and $r_{0}=\max \left\{v_{1}, v_{3}\right\}$ give the smallest $r_{0}$ that satisfies all three constraints. Taking the scale factors into account give the optimal solution

$$
r_{0}^{\star}=\frac{\gamma \lambda_{e} \kappa}{N} \max \left\{v_{1}, v_{3}\right\}, \quad r_{1}^{\star}=\frac{\gamma \lambda_{e} \kappa}{N} v_{2} .
$$

Here can we see that tighter specification (larger $\gamma$ ), higher noise variance (larger $\lambda_{e}$ ) and higher probability (larger $\kappa$ ) all require more input power, while increasing the number of data point $N$ reduces the required power.

We can also compare the optimal solution $r_{0}^{\star}$ with the power needed for a white noise input signal. We then need to solve

$$
\min _{r_{0}} r_{0} \quad \text { s.t } \quad \frac{N}{\lambda_{e} \kappa}\left[\begin{array}{cc}
r_{0} & 0 \\
0 & r_{0}
\end{array}\right] \geq \gamma V_{a p p}^{\prime \prime}\left(\theta_{o}\right),
$$

with solution

$$
r_{0}^{w}=\frac{\gamma \lambda_{e} \kappa}{N} \max \operatorname{eig}\left\{V_{a p p}^{\prime \prime}\left(\theta_{o}\right)\right\}
$$

where the maximum eigenvalue of the Hessian, (19), equals

$$
\begin{aligned}
& \max \operatorname{eig}\left\{V_{a p p}^{\prime \prime}\left(\theta_{o}\right)\right\}=0.5\left[v_{1}+v_{3}+\sqrt{\left(v_{1}-v_{3}\right)^{2}+4 v_{2}^{2}}\right] \text {, } \\
& v_{1}, v_{3} \geq 0,\left(v_{1} v_{3}\right) \geq v_{2}^{2} \text {. }
\end{aligned}
$$

For fixed $v_{1}$ and $v_{3}$ the maximum eigenvalue is maximized with respect to $v_{2}$ for $v_{2}^{2}=\left(v_{1} v_{3}\right)$. We then have $\max \operatorname{eig}\left\{V_{a p p}^{\prime \prime}\left(\theta_{o}\right)\right\}=v_{1}+v_{3}$. This should be compared with $\max \left(v_{1}, v_{3}\right)$ for the optimal signal (20). Hence we can gain up to a factor of two in input power by using the optimal input compared with white noise.

\section{A. Time Realizations}

There are in principle two direct ways to find a time realization $\{u(t)\}$ for a given covariance sequence. The first one is to use an AutoRegressive (AR) process and the second one is to use sinusoidal signals in white noise (the Carathéodory parametrization). For example, assume the input is generated by the AR process $u(t)=-a u(t-1)+e_{u}(t)$, where $\left\{e_{u}(t)\right\}$ is white noise with variance $\lambda_{u}$. Take

$$
a=-\frac{r_{1}^{\star}}{r_{0}^{\star}}, \lambda_{u}=\frac{\left(r_{0}^{\star}\right)^{2}-\left(r_{1}^{\star}\right)^{2}}{r_{0}^{\star}} .
$$

then

$$
\mathrm{E}\left\{u^{2}(t)\right\}=\frac{\lambda_{u}}{1-a^{2}}=r_{0}^{\star}, \quad \mathrm{E}\{u(t) u(t-1)\}=\frac{-a \lambda_{u}}{1-a^{2}}=r_{1}^{\star} .
$$

\section{B. Control Example, Revisited}

Reconsider the P-controller example in Section II-A, for which

$$
V_{a p p}^{\prime \prime}\left(\theta_{o}\right)=2\left[F^{\prime}\left(\beta b_{1}^{o}-b_{2}^{o}\right)\right]^{2}\left[\begin{array}{cc}
\beta^{2} & -\beta \\
-\beta & 1
\end{array}\right], \quad 0<\beta<1 .
$$

For this application the optimal solution to (18) is

$$
r_{0}^{\star}=\frac{2 \gamma\left[F^{\prime}\left(\beta b_{1}^{o}-b_{2}^{o}\right)\right]^{2} \lambda_{e} \kappa}{N}, \quad r_{1}^{\star}=-\beta r_{0}^{\star}
$$

We notice that $2 \gamma\left[F^{\prime}\left(\beta b_{1}^{o}-b_{2}^{o}\right)\right]^{2}$ is the relevant measure for the "size" of the application set. If we enforce $r_{1}=0$, we need to take $r_{0} \geq\left(1+\beta^{2}\right) r_{0}^{\star}$. It is interesting to notice that we can gain up to a factor of two in power if we instead use the optimal solution. An alternative is to reduce the experimental time with up to a factor of two by using the optimal input signal. 


\section{A Model Predictive Control Example}

The final example concerns Model Predictive Control. Here it is not possible to analytically find $V_{a p p}^{\prime \prime}(\theta)$, and we will use numerical differentiation to determine $V_{a p p}^{\prime \prime}\left(\theta_{o}\right)$. Consider the FIR state space model

$$
\begin{aligned}
{\left[\begin{array}{l}
x_{1}(t+1) \\
x_{2}(t+1)
\end{array}\right] } & =\left[\begin{array}{ll}
0 & 0 \\
1 & 0
\end{array}\right]\left[\begin{array}{l}
x_{1}(t) \\
x_{2}(t)
\end{array}\right]+\left[\begin{array}{l}
1 \\
0
\end{array}\right] u(t) \\
y(t) & =\left[\begin{array}{ll}
b_{1} & b_{2}
\end{array}\right]\left[\begin{array}{l}
x_{1}(t) \\
x_{2}(t)
\end{array}\right]+d(t),
\end{aligned}
$$

where the state vector contains delayed inputs. The disturbance $d(t)$ is equal to one, $t \geq 0$, and we assume zero initial conditions. The disturbance is assumed to be unknown in the MPC algorithm, so it will be estimated for future $t$. The feed-forward open loop control

$$
u(t)=\frac{-d(t)}{b_{1}+b_{2}}
$$

rejects the disturbance in two time steps. We will study the finite horizon quadratic cost function

$$
V_{\text {control }}(u)=\sum_{t=1}^{T-1} y^{2}(t)
$$

subject to the constraint $|u(t)| \leq u_{\max }$, and implement a receding horizon control strategy. We need $u_{\text {max }} \geq$ $|d| /\left|b_{1}+b_{2}\right| \mid$ to avoid stationary errors. The application cost function is

$$
V_{a p p}(\theta)=\frac{1}{M} \sum_{t=1}^{M}\left[y(t, \theta)-y\left(t, \theta_{o}\right)\right]^{2},
$$

where $y(t, \theta)$ is the output signal under the MPC feedback control based on the model $\theta$. The application set $\mathscr{E}_{a p p}$ is defined by (6), and system identification set $\mathscr{E}_{S I}$ is defined by (7).

We will first study systems with the zero inside the unit disc, $\left|b_{2}^{0} / b_{1}^{0}\right|<1$. The true parameters are $b_{1}^{0}=10$ and $b_{2}^{0}=-9$, i.e., the zero is at 0.9 and the static gain is 1 . The MPC prediction horizon is $T=10$, and $V_{a p p}(\theta)$ is calculated with $M=10$. To start with there is no constraint on the input signal, $u_{\max }=\infty$, in the MPC control problem. The corresponding MPC problem is solved in MATLAB by using $\mathrm{CVX}$, a package for specifying and solving convex programs [11], [10]. The Hessian $V_{a p p}^{\prime \prime}\left(\theta_{0}\right)$ is found using numerical differentiation and Problem (18) is then solved with respect to $r_{0}$ and $r_{1}$ using (20) with $\gamma=50, \lambda_{e}=10^{-2}, N=100$ and $\kappa$ from the upper $5 \%$ percentile $(\alpha=0.95)$ of the $\chi^{2}(2)$ distribution. The optimal input signal is used in calculating the system identification ellipsoidal set. Figure 1 shows the ellipsoidal sets. The system identification ellipsoid is inside the application one, which means that estimated model will with at least $95 \%$ probability satisfy the application specification.

The eigenvector corresponding to the larger semi-axis of $\mathscr{E}_{\text {Vapp }}$ in Figure 1 is $[0.70,-0.72]^{\mathrm{T}}$ with eigenvalue 0.002 . The larger eigenvalue is 0.062 with the corresponding eigenvector $[-0.72,-0.70]^{\mathrm{T}}$. This means that it is most important to have a good estimate of the sum of the parameters, i.e., the static gain should be close to that of the true system. This makes sense since we do not put any constraint on $u(t)$ and the feed forward solution (25) is close to optimal.

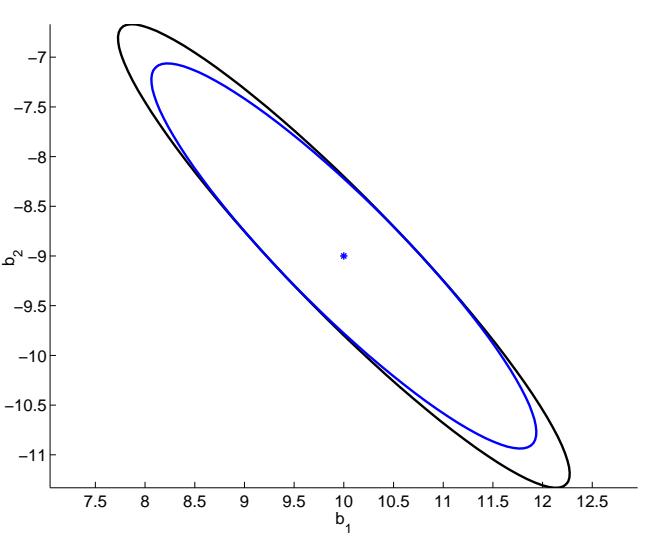

Fig. 1. $\mathscr{E}_{a p p}$ is the outer ellipse and $\mathscr{E}_{S I}$ is the inner ellipse.

Figure 2 shows $\hat{\theta}_{N}$ when the optimal input signal is used in the system identification experiment with zero mean white Gaussian noise $e(t)$ with variance $\lambda_{e}=10^{-2}$ and no disturbance $d(t)$. One thousand $\hat{\theta}_{N}$ are estimated by applying the least-squares method to one hundred measurements of $u(t)$ and $y(t)$. In total $95.7 \%$ of the estimated parameters are inside the ellipse $\mathscr{E}_{S I}$.

The level curve of $V_{a p p}(\theta)=1 /(2 \gamma), \gamma=500$, is given in Figure 3 , along with its approximation $\mathscr{E}_{\text {app }}$. We see some discrepancy in the center point, but the basic geometry is captured by the ellipsoidal approximation . A higher accuracy $\gamma$ will lead to a better approximation of $V_{a p p}(\theta)$.

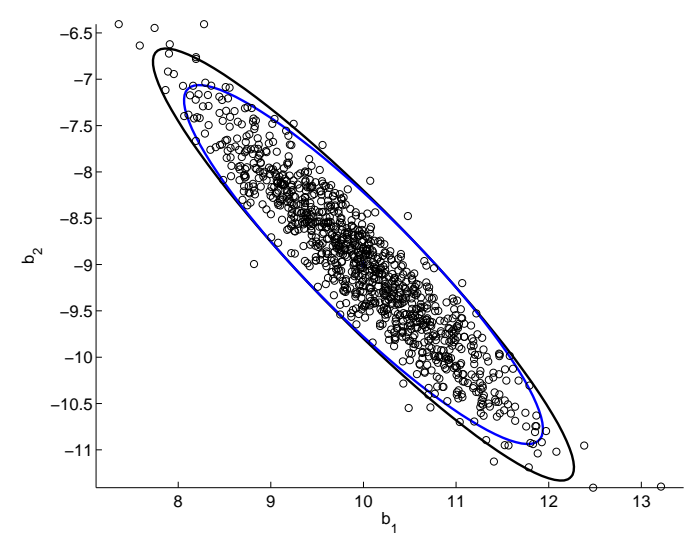

Fig. 2. $\mathscr{E}_{a p p}$ is the outer ellipse, $\mathscr{E}_{S I}$ is the inner ellipse and $\hat{\theta}_{N}$ are the small circles.

To obtain a more difficult control problem consider a system with a zero outside the unit circle, $\theta_{0}=[-5,10]^{\mathrm{T}}$. The static gain is 5 and the zero is at -2 . We set the prediction horizon to $T=20$, the number of samples in $V_{a p p}(\theta)$ to $M=20$ and restricting the amplitude of the input signal, $|u(t)| \leq u_{\max }=1$. We need $u_{\max } \geq 0.2$ to avoid static errors. Figure 4 shows the corresponding ellipsoids. Notice that the direction that tolerates larger errors in the parameter estimates has shifted to $[-0.94,0.35]$, and that it is here difficult to find a large system identification set that is inside the application set. The reason is the difference in possible eigen-vector directions. This example highlights the 


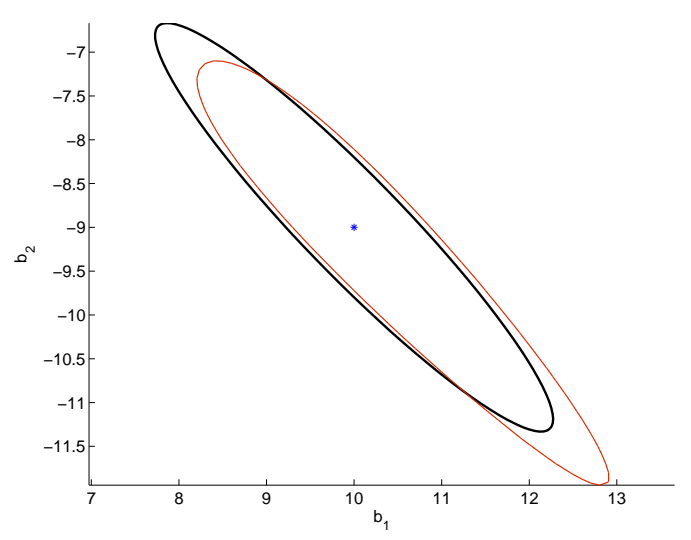

Fig. 3. $\mathscr{E}_{a p p}$ is the upper ellipse and the level curve corresponding to $V_{\text {app }}(\theta)=1 /(2 \gamma), \gamma=50$ is the lower contour.

importance of understanding how the intended application of the estimated model affects the system identification experiment, and in particular the choice of experiential input signal.

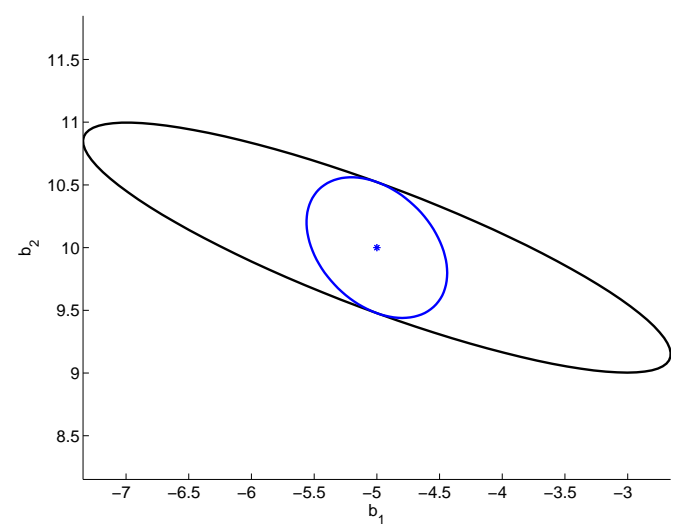

Fig. 4. $\mathscr{E}_{a p p}$ is the outer ellipse and $\mathscr{E}_{S I}$ is the inner ellipse.

\section{CONCLUSION}

The system identification input design approach developed in [13] directly relates the experiment design to the intended application of the model. Our objective has been to illustrate and evaluate this framework on some basic control problems. Future work include development of software tools for optimization based system identification experiment design for more complex MPC problems, and to evaluate the results in industrial control applications.

\section{REFERENCES}

[1] M. Barenthin, X. Bombois, H. Hjalmarsson, and G. Scorletti. Identification for control of multivariable systems: Controller validation and experiment design via LMIs. Automatica, 44(12):3070-3078, 2008.

[2] M. Barenthin, H. Jansson, H. Hjalmarsson, J. Mårtensson, and B. Wahlberg. A control perspective on optimal input design in system identification. In Forever Ljung in System Identification, chapter 10. Studentlitteratur, September 2006

[3] G. Belforte and P. Gay. Optimal experiment design for regression polynomial models identification. International Journal of Control, 75(15):1178-1189, 2002.
[4] X. Bombois, G. Scorletti, M. Gevers, P. M. J. Van den Hof, and R. Hildebrand. Least costly identification experiment for control. Automatica, 42(10):1651-1662, 2006.

[5] U. Forssell and L. Ljung. Some results on optimal experiment design. Automatica, 36(5):749-756, May 2000.

[6] Gaia Franceschini and Sandro Macchietto. Model-based design of experiments for parameter precision: State of the art. Chemical Engineering Science, 63(19):4846 - 4872, 2008.

[7] F. R. Gantmacher. The theory of matrices. Volymes: 1, 2. Chelsea Publishing, Co., New York, 1959.

[8] L. Gerencsér and H. Hjalmarsson. Identification of ARX systems with non-stationary inputs - asymptotic analysis with application to adaptive input design. Automatica, 45(3):623-633, March 2009.

[9] M. Gevers, L. Miskovic, D. Bonvin, and A. Karimi. Identification of multi-input systems: variance analysis and input design issues. Automatica, 42(4):559-572, 2006.

[10] M. Grant and S. Boyd. Graph implementations for nonsmooth convex programs. In V. Blondel, S. Boyd, and H. Kimura, editors, Recent Advances in Learning and Control (a tribute to M. Vidyasagar), pages 95-110. Lecture Notes in Control and Information Sciences, Springer, 2008.

[11] M. Grant and S. Boyd. Cvx: Matlab software for disciplined convex programming (web page and software). http://stanford.edu/ royd/cvx, June 2009.

[12] R. Hildebrand and M. Gevers. Identification for control: Optimal input design with respect to a worst-case $v$-gap cost function. SIAM J. Control Optim, 41(5):1586-1608, 2003.

[13] H. Hjalmarsson. System identification of complex and structured systems. European Journal of Control, pages 275-2310, 2009.

[14] H. Hjalmarsson and H. Jansson. Closed loop experiment design for linear time invariant dynamical systems via LMIs. Automatica, 44(3):623-636, 2008.

[15] Håkan Hjalmarsson. From experiment design to closed loop control. Automatica, 41(3):393-438, March 2005.

[16] H. Jansson and H. Hjalmarsson. Input design via LMIs admitting frequency-wise model specifications in confidence regions. IEEE Transactions on Automatic Control, 50(10):1534-1549, 2005.

[17] C. Jauberthie, L. Denis-Vidal, P. Coton, and G. Joly-Blanchard. An optimal input design procedure. Automatica, 42(5):881 - 884, 2006.

[18] K. Lindqvist and H. Hjalmarsson. Optimal input design using linear matrix inequalities. In IFAC Symposium on System Identification, 2000 .

[19] L. Ljung. Asymptotic variance expressions for identified black-box expressions for identified black-box transfer functions models. IEEE Transactions on Automatic Control, AC-30:834-844, 1985.

[20] L. Ljung. System Identification - Theory For the User, 2nd ed. Prentice Hall, Upper Saddle River, New Jersey, 1999.

[21] B. Ninness and S. Gibson. Quantifying the accuracy of Hammerstein model estimation. Automatica, 38:2037-2051, 2002.

[22] L. Pronzato. Adaptive optimization and D-optimum experimental design. The Annals of Statistics, 28(6):1743-1761, 2000.

[23] L. Pronzato. Optimal experimental design and some related control problems. Automatica, 44(2):303-325, February 2008

[24] C. R. Rojas, J. C. Agüero, J. S. Welsh, and G. C. Goodwin. On the equivalence of least costly and traditional experiment design for control. Automatica, 44(11):2706-2715, 2008.

[25] C. R Rojas, J. S. Welsh, and J. C. Agüero. Fundamental limitations on the variance of parametric models. IEEE Transactions on Automatic Control, pages 1077-1081, 2009.

[26] C. R Rojas, J. S. Welsh, G. C. Goodwin, and A. Feuer. Robust optimal experiment design for system identification. Automatica, 43(6):9931008, June 2007. 